\title{
HYBRID STRUCTURAL SKIN: PROTOTYPE OF A GFRP ELASTIC GRIDSHELL BRACED BY A FIBER-REINFORCED CONCRETE ENVELOPE
}

\author{
Pierre Cuvilliers ${ }^{a, b}$, Cyril Douthe ${ }^{a}$, Lionel du Peloux ${ }^{a}$, Robert Le Roy c,a \\ a Université Paris-Est, Laboratoire Navier (UMR 8205), ENPC, IFSTTAR, CNRS, Marne-La-Vallée, France. \\ b (Present) Building Technology, Department of Architecture, Massachusetts Institute of Technology, Cambridge, MA, USA. \\ C Laboratoire GSA, ENSAPM, Paris France \\ pcuvil@mit.edu, lionel.dupeloux@gmail.com, cyril.douthe@ifsttar.fr, robert.leroy@enpc.fr
}

Editor's Note: This space reserved for the Editor to give such information as date of receipt of manuscript, date of receipt of revisions (if any), and date of acceptance of paper. In addition, a statement about possible written discussion is appended.

DOI: Digital Object Identifier to be provided by Editor when assigned upon publication

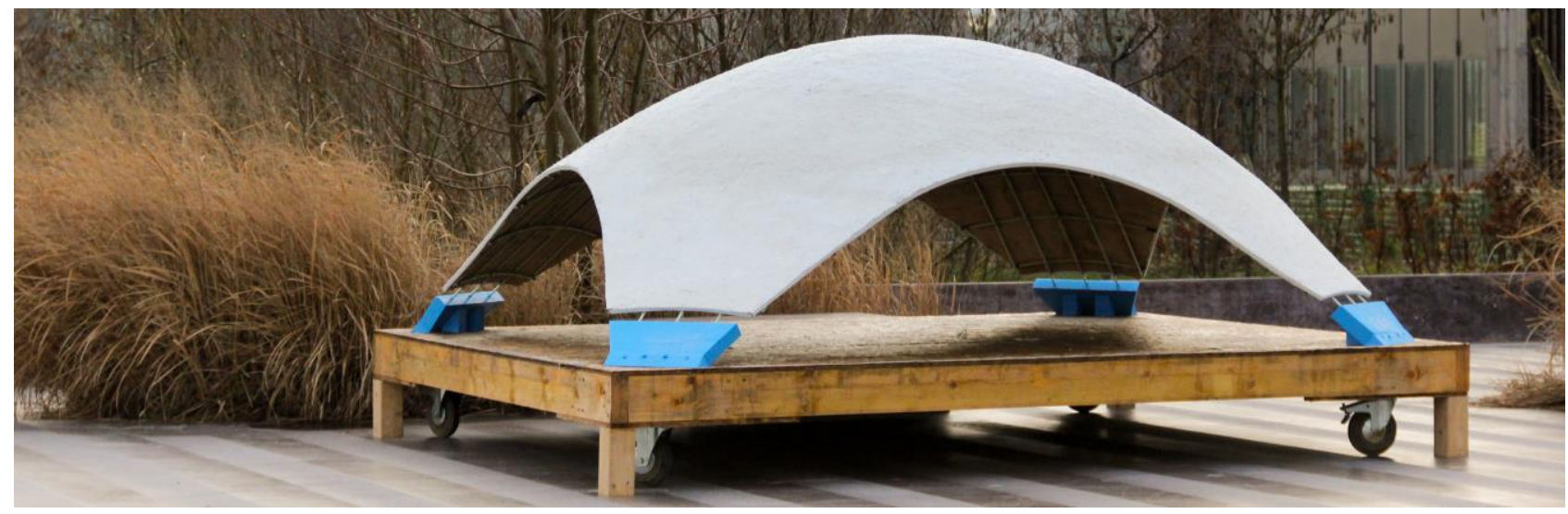

Figure 1: 3-by-3-meters prototype of GFRP gridshell braced by a concrete envelope built at the Ecole des Ponts ParisTech.

\section{ABSTRACT}

The simulation and construction of elastic gridshells in composite materials is nowadays a technique that one can consider mastered [1]. The bracing of the grid in its final form remains however a time consuming step with a lot of manual work. The lack of alternatives to membrane covering is also an important limitation to the development of such technology. The proposed paper tries to tackle both issues through a novel concept of a hybrid structural skin made of an elastic gridshell braced with a concrete envelope. The idea is to use the gridshell as a formwork for the concrete and to guarantee a mechanical connection between the thin concrete skin and the main grid, so that the concrete ensures the bracing of the grid and that the thickness of the concrete is reduced to a minimum. To demonstrate the feasibility and interest of this structural concept, a $10 \mathrm{~m}^{2}$ prototype has been built. The main aspects of the design and of the realization of the prototype are presented here (including detailing and mix design).

Keywords: Structural morphology, Lightweight structures, Soft-formwork, conceptual design

\section{INTRODUCTION}

Elastic gridshells or strained gridshells are an efficient solution to temporarily cover large spaces without intermediary supports. Their main advantages are the speed at which they can be installed, and their ratio of weight to covered area: $300 \mathrm{~m}^{2}$ gridshells have been installed and covered in a few days, weighing approximately $50 \mathrm{daN} / \mathrm{m}^{2}$ (Solidays forum, France, 2011 [2] or the Créteil' provisory cathedral, France 2013 shown in Figure 2 [1]). They have been built successfully from diverse materials such as wood (Weald \& Downland museum, UK, 2002 [3] or the Mannheim multihalle, Germany, 1975, still in use forty years after its original two years commissioned life-span [4]), 
cardboard (Japan pavilion at Expo 2000, Germany [5]) and fiberglass composite material [1], [2]. Elastic gridshells are generally made out of a flat regular grid of long rods, connected at each intersection by a node allowing rotation in the plane of the grid. This grid is then deformed into a 3D shape (by pushing or hoisting it) and pinned on its boundary to the ground. This elastic process is possible because the nodes allow shear movements in the grid. The resulting surface is usually rigidified in a later step by adding a layer of cables [5], [6] or rigid diagonal members bracing the grid [1], [2], [7] or by inserting rigid cladding panels in the rhomboid cells [8]. The as-built shape obtained is the result of the bending equilibrium of the rods in large displacements.

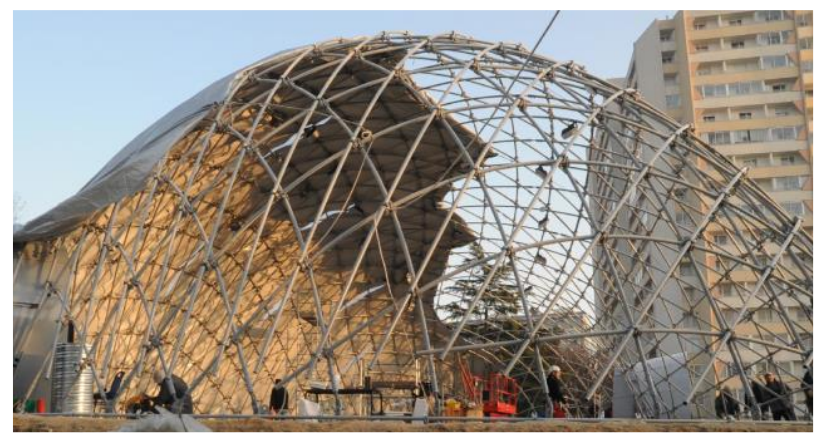

Figure 2: Créteil gridshell, covering in progress.

Covering this type of structure is difficult for several reasons. First, predicting the final shape is difficult: it involves advanced beam theory [9] and is very sensitive to small perturbations such as boundary conditions and nodal eccentricity [10]. Thus, many gridshells have been covered using soft fabrics to avoid the need for an accurate a priori knowledge of the required shape of cladding panels. Second, the natural quadrangular cells of the structure offer few regularity properties that would help create an efficient cladding system directly supported at the nodes. Therefore hard covers on elastic gridshells are often offset away from the gridshell to create the geometric properties needed (like for the Downland museum [3] or for the Chiddingstone Orangery [11]).

Third, since the gridshell often combines the function of a roof and a wall, it needs to accommodate diverse envelope conditions; mainly light transmission and insulation. Soft fabrics for example easily let light through, but are very poor insulation capabilities. Lastly, as the complexity of the roof system increases, the nodes will grow in size, weight and construction time. Since they are repeated so many times, this has the potential of becoming a crippling component in the structure.

This project investigates the feasibility of a new kind of structural shell. It combines an elastic gridshell with a bracing fiber-reinforced concrete envelope to realize a rigid roof system of limited weight, longspan capabilities, with no additional scaffolding, and limitless forms. The paper is organized as follows. In Section 2, we look at the possible and existing relationships between an elastic gridshell and its envelope, and show that there is potential for new developments in hard-covered gridshells, especially as flexible formwork for concrete shells. We then restrict the study to the interaction of concrete envelops with elastic gridshells, and present the arguments for the structural and constructive principles experimented in our prototype. Section 3 lays out the steps of the design process of the 3-by-3 meters prototype and its construction process. Section 4 finally discusses the results and looks at possible larger scale realizations.

\section{CONSTRUCTION TECHNIQUES FOR GRIDSHELLS AND CONCRETE SHELLS}

After the unbraced grid is assembled and bent, giving the structure its natural shape, two necessary steps remain that are often time-consuming. First, the bracing of the initial grid which will give it its shell behavior by rigidifying shear movements and collecting the associated shear forces. Second, the covering which will turn the structure into an enclosed space. Both steps currently require working on each node or cell of the structure while it is in its final shape - which is harder than on the ground - and possibly not yet rigidified. Additionally, most of the time (except for the Savill building [8]), these two steps are separate processes that do not benefit from each other.

\subsection{Existing bracing methods}

Three methods to confer shear stiffness to a gridshell are typically considered in elastic gridshell design: rotation-locking at nodes, diagonal bracing by cables or with rigid members, rigid panels. These three methods will be described below and we will show that conceptual developments for elastic gridshells bracing are most accessible in strategies similar to the rigid panels, where bracing and envelope are joined. 
Rotation-lock at nodes is mentioned negatively in $[7,8]$; in Mannheim, the clamping capacity of the connection was tested experimentally but found insufficient so that it was completed by cables. Grafe et al. in [12] reference a few unbuilt projects, mostly with metallic members, that brace the gridshell by node-locking. Apart for the Mannheim gridshell, the only built elastic gridshell with rotation-locked nodes referenced in the literature is one of the first experiments on elastic gridshells by Frei Otto, at Berkeley in 1962, in the same book [13]. The problem with this strategy is that it creates two largely diverging design objectives on the node: it should be completely free in large rotations during the shape erection, and after that as rigid as possible in small rotations. Thus the resulting node is expensive, either in mechanical construction or in modification time when it is locked. Moreover, generally speaking clamping nodes is not the most efficient strategy to brace a frame, so that we do not further consider possible improvements in elastic gridshells based on this kind of nodes.

Diagonal cable bracing has been used in the Mannheim multihalle [14] or for the Chiddingstone Orangery [11], for example. The main advantage of this method is its simplicity, as small appendices on nodes will often be enough to attach the cables, and on-site operations do not require large tools. Two orthogonal directions of bracing are needed with cables, since they only have stiffness in tension. Diagonal rigid bracing is very similar, except that it requires bigger adaptations on the nodes. However, the on-site operations can be reduced since only one direction of bracing is needed without any recourse to prestressing. For example, du Peloux et al. in the Créteil Cathedral [1] use a third direction of continuous members with same diameter as the main grid while D'Amico et al. [15] use short trusses in each cell. Both strategies have matured with the developments of elastic gridshells and are largely used in their construction today. Therefore we did not focus on these bracing strategies either (which does not mean that new conceptual developments cannot be expected following for example the interesting case-study on segment lath modular grid by Mork et al [16]).

Panel bracing combines the roles of a rigid envelope and bracing of the shell by bolting plywood panels to the laths. The Savill garden gridshell [8] used this technique to clad its wooden gridshell. This collaborative strategy between envelope and bracing allowed the engineers of the project to achieve a smooth and insulated roof finish, directly on top of the bracing. This shows that surface bracing methods have the potential to help reduce the total cost of a gridshell building because they can also act as a solid cover, which is interesting as we show in the next section.

\subsection{Existing covering methods}

In addition to bracing, gridshell structures need a second operation for their final shape to become a building: covering. This operation supposes finding a material and a technique that will be able to adapt to the complex shape of the gridshell. Common covering solutions can be separated into two categories, based on the local surface bending stiffness of the finished product: soft and hard covers. Here we show why hard covers are desirable but difficult to design.

Soft covers have been designed in fabrics with PVC coating, for example for the Mannheim multihalle and the Créteil cathedral, or in paper for the Japan Pavilion. They have the advantage of being easy to install on freeform shapes, and lightweight so that they do not add weight on the structure. However they are prone to tearing and have very limited ability to support additional loads such as insulation or cleaning personnel, so they are better suited to temporary structures.

Hard covers on the contrary can have a good secondary structural capability but are harder to design and build. Traditional carpentry methods for double curved envelopes have existed for centuries (think about ships' hulls or Paris zinc roofs), approximating the surface by small developable strips. Interesting examples of application to gridshells include a complex of plywood, insulation, zinc standing-seam skin and oak rain-screen at the Savill garden gridshell [8]; polycarbonate and RoofKreete ${ }^{\circledR}$ at the Downland gridshell [7]; and a frameless triangulated glass cover over the Chiddingstone Orangery gridshell [11]. There is however some contradiction in the simplicity of the grid construction and the skills required for realization of these covers. Recently a promising solution for generating equilibrium shapes in the vicinity of PQ-Meshes offered a perspective for the covering of elastic gridshells with quadrangular planar facets [17], but, for the moment, no realization has been made. Still, all these covers introduce in the 
design of the connections complex new constraints which necessarily increase the total thickness of the structure.

It will thus be interesting to investigate a covering method for elastic gridshells that combines the ease of design and construction of a soft cover with the resistance of a hard cover.

\subsection{Concrete shells and scaffolding}

Recently, there was a renewal of interest in research on thin concrete shells cast on soft formworks, mostly fabric and cable nets [18]-[20]. It was shown to be a cost-efficient and labor-saving method to build concrete shells of complex forms. The fabric formwork is also both lightweight and, when reinforced with a cable net, strong. Then, the scaffolding needed can be reduced to the edges of the structures. Using an elastic gridshell to support a fabric formwork could interestingly build on this idea of a lightweight scaffolding spanning from edge to edge. It would also enable the realization of forms of both positive and negative Gaussian curvature, something that is not possible in the cable-net and fabric formwork of [20]. However, removing the gridshell after pouring the concrete seems both complicated and unneeded. We can instead use this structure, and create a collaboration, a composite action between the two materials. This idea is developed in more details in the following section.

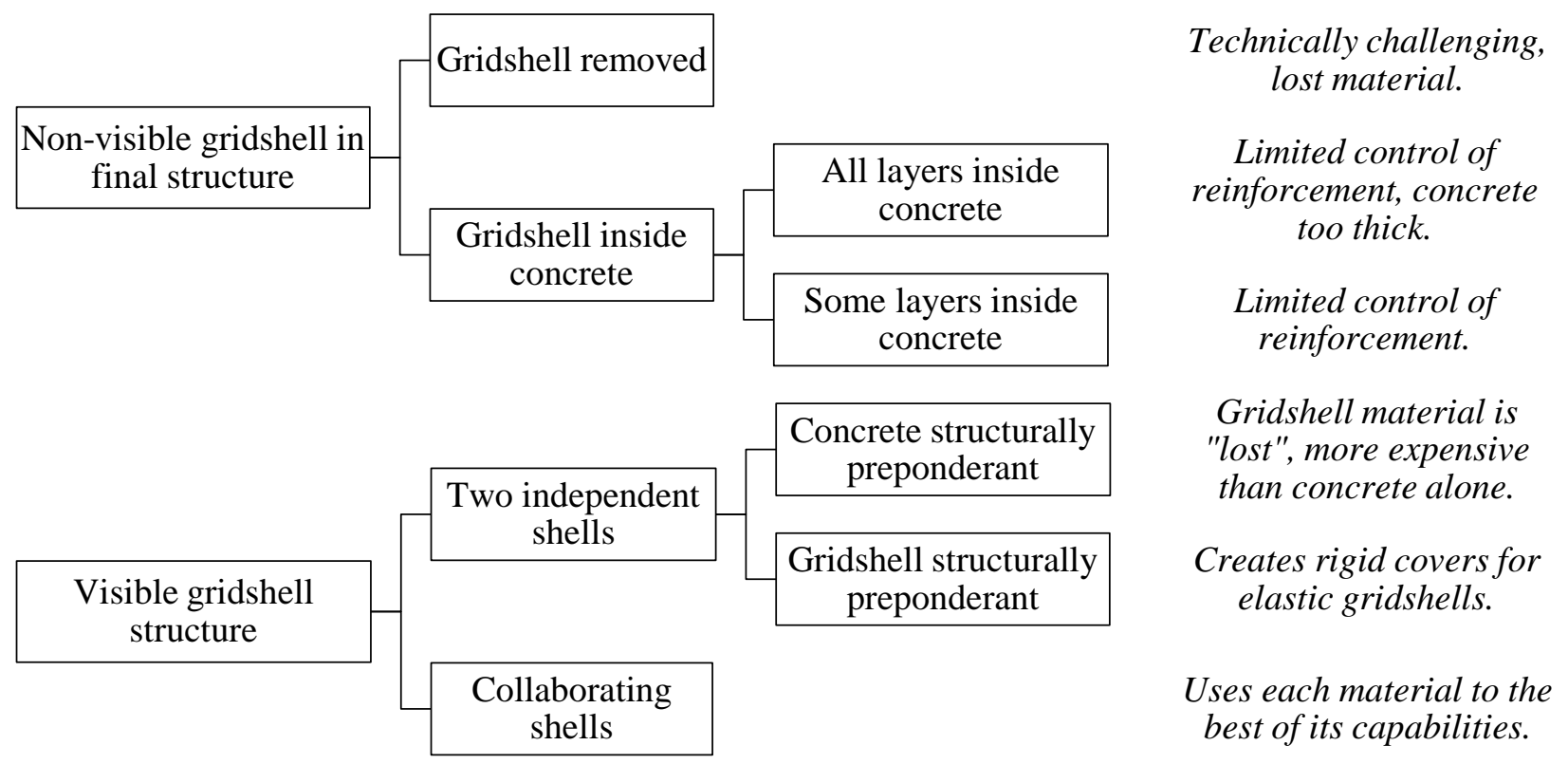

Figure 3: Taxonomy of the possible interactions between an elastic gridshell and a concrete layer.

\subsection{Interactions between an elastic gridshell and a concrete skin}

Given the lack of precedent for the use of elastic gridshells in concrete structures, we investigated the different modes of interactions between an elastic gridshell and a concrete skin poured on or over it. They are summarized in Figure 3. The conceptual evaluation of each possibility is done with a domelike structure in mind, where loads are dominated by gravity and preferably resisted by membrane stresses.

The first distinction we make is on the visibility of the gridshell in the finished structure. This is mainly an architectural decision but it also influences the thickness of the concrete layer. If the gridshell is not to be seen in the finished structure, it has to be removed when the concrete has cured or the concrete has to be poured completely around it.

i) Removing the gridshell was deemed to be technically challenging: the removing of the centering in vault structures is known to be one of the most delicate moves. Besides, the projected area of the flat grid being always larger than that of the deformed one, a way of splitting the grid, allowing to progressively relax the bent rods and let them straighten again to slide under the dome must be designed. Nevertheless with this 
technique, one could imagine reusing the dismounted grid as a new formwork for another concrete shell with similar or totally different form.

ii) Pouring the concrete around the gridshell seems promising at first, as it could use the gridshell as a reinforcement. However, it requires a very thick concrete layer to cover the gridshell: in our example, the combined thickness of the two layers of GFRP rods is $20 \mathrm{~mm}$. With a padding of around $15 \mathrm{~mm}$ on both sides to accommodate realistic construction constraints, we get a $50 \mathrm{~mm}$ concrete layer with a thickness over span ratio of $1 / 40$. This compares badly to the typical ratio of $1 / 500$ for a reinforced concrete shell [21, p. 35], [22, p. 185]. More importantly, the weight of the wet concrete would probably exceed the capacity of the gridshell, so that it would be necessary to install provisory additional supports which reduce the simplicity of the method. Furthermore, we anticipate a limited efficiency of the gridshell as a reinforcement as its geometry will first have to obey to a different rationale of supporting the fresh concrete.

If the gridshell is visible in the final structure, we can either have the gridshell structure and the concrete shell standing up as two independent structures, or as one mixed shell. With two independent structures, some material is once again structurally inefficient. If the concrete is a lot stiffer than the gridshell, then the structure built is a concrete shell with a grid hanging below it; the main interest of this being in using the gridshell as a scaffolding. If the gridshell is a lot stiffer than the concrete, we created a smooth and continuous rigid cover for elastic gridshells which will deform under loading with a high risk of cracking of the concrete.

Both solutions (the gridshell as a formwork for a concrete shell and the rigid cover) were in our initial objectives for this study and are interesting research directions on their own, but it seems that a hybrid structural skin could beneficially combine all advantages. By applying a skin of concrete on top of an elastic gridshell and connecting it mechanically to the main grid, we will create a rigid, continuous and curved envelop that improves the structural capabilities of the gridshell. The intention is indeed that the composite action of the grid and the concrete skin insures bracing of the grid, redistribution of stresses under concentrated load and reduction of local buckling. The feasibility of this concept is demonstrated in the rest of the article.

\section{PROOF OF CONCEPT}

\subsection{Design objectives of the prototype and general construction process}

Based on the conclusions of Section 2, we describe in this section the design methodology for a prototype of an elastic gridshell braced by a rigid concrete envelope. The shape of the structure results from two different sets of constraints: constraints on the shape of the elastic gridshell, and funicularity constraints. To find an interesting solution to those constraints, an iterative form-finding process was used. It consists in six successive steps implemented in the parametric environment Grasshopper ${ }^{\circledR}$ for Rhinoceros $^{\circledR}$. The succession of the steps is represented in Figure 4 and detailed in the following:

i) Define a contour where the gridshell will be anchored and choose an approximate region where the concrete will be placed;

ii) Generate a target funicular shape from this concrete and the inverted concrete load using the stress density method [23], [24];

iii) Define a Chebyshev net on this shape using the compass method [25], [26] and then find the equilibrium of this gridshell under self-weight using Dynamic Relaxation [27], [28];

iv) Analyze the shape of this gridshell when loaded with wet concrete, and check its stability with dynamic relaxation again;

v) Remove the lateral parts of the shell so that bending is minimized around the openings;

vi) Verify the stresses in the obtained composite shell under load. The finite element analysis is conducted neglecting the prestress in the members (which has been shown to have limited consequences on the behavior [29]) and considering elastic connection between the concrete and the grid. Long term effects are not taken into account. 

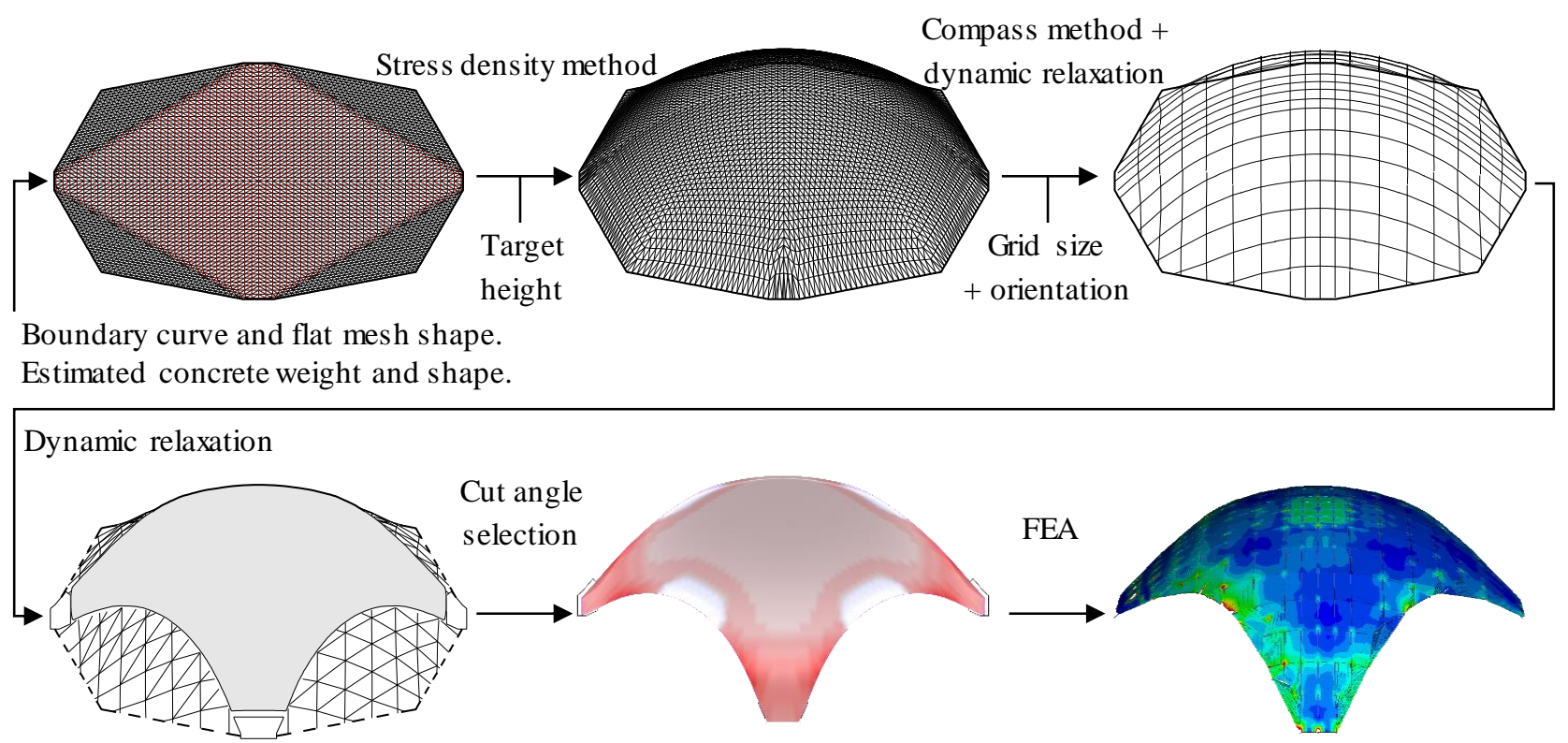

Figure 4: Steps of the design process used for the prototype.

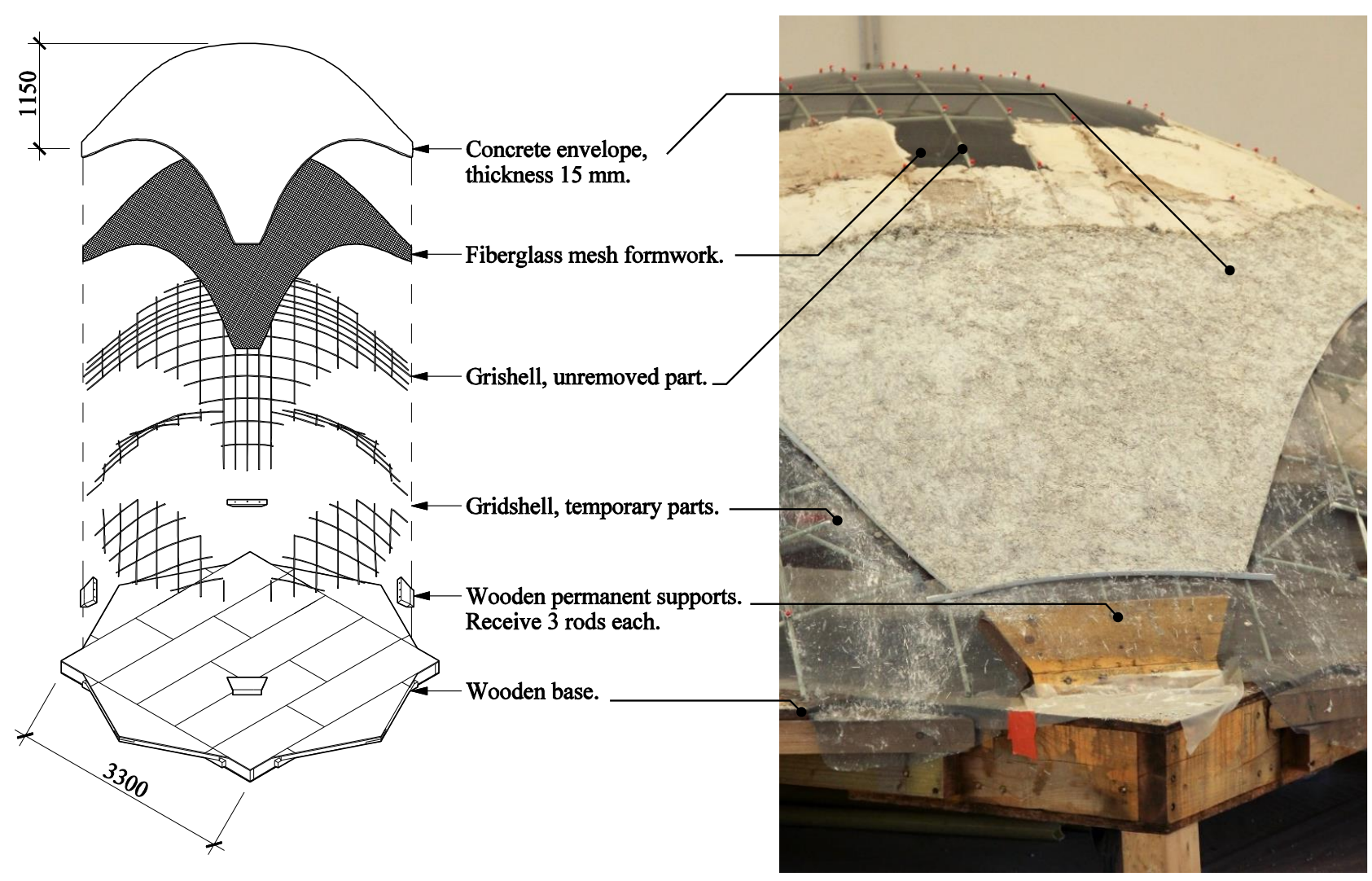

Figure 5: Axonometric view and photograph showing the successive layers in the prototype. 


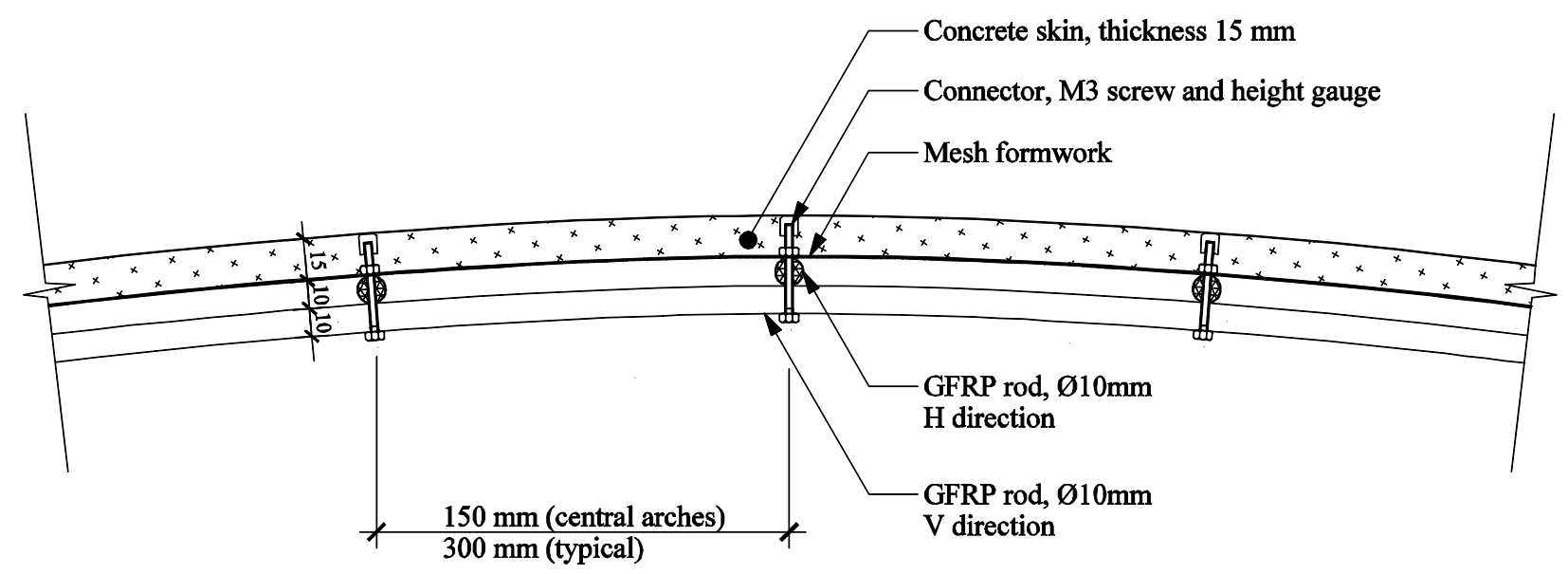

Figure 6: Section cut, typical.

\subsection{Geometrical and mechanical characteristics of the prototype}

Following the methodology presented above, the prototype presented in Figure 5 in axonometric view was built on the campus site. Two components are of particular importance: the fiberglass mesh fabric that provides the formwork for the concrete, and the screw connector that binds the concrete and the gridshell layer, which can be seen in the typical detail section of Figure 6.

Figure 5 shows the succession of layers of the structure. On a wooden support structure, an elastic gridshell is shaped, initially with no openings: the whole boundary of the gridshell is attached to the ground. This support structure is temporarily partially triangulated with cables (not shown on the drawings). A fiberglass mesh is laid on the gridshell on the area that will receive the concrete. This mesh is a woven fabric of relatively thick fiberglass threads, similar to what is used for insect screens on windows. The concrete is introduced last; it does not cover the whole gridshell so that the structure does not remain as a complete dome in the end. When it has cured, the parts of the gridshell that are not covered by concrete are removed, creating four openings. The structure is $1.15 \mathrm{~m}$ high with openings $0.7 \mathrm{~m}$ high. The structure spans $3 \mathrm{~m}$ from support to support. Those four supports are placed at each corner of the gridshell. The developed area of the concrete layer is $9.55 \mathrm{~m}^{2}$ and the structure covers $8.47 \mathrm{~m}^{2}$ in plan. With these proportions, the height over span ratio of the shell stands in the average range of most built concrete shells [30].
At the permanent supports, to limit as much as possible the moments induced by edge fixities, we chose to fix only the elastic gridshell, creating elastic hinges supports for the structure. The concrete envelope stops a few centimeters above the supports and remains stress free on the edge. The load transfer is ensured from the concrete to the gridshell all along the structure by the connectors: this is the purpose of mixed action. This small space between the envelope and the foundation is intended at visually demonstrating that the elastic gridshell is a central structural component of the hybrid shell roof, which cannot be considered has a simple concrete shell with a gridshell formwork. There was however an important drawback with this technical solution: in the vicinity of the supports, the grid members were parallels and without bracing on a distance of approximately $8 \mathrm{~cm}$, so that the built prototype is subject to a rotational instability and has a limited bearing capacity.

Figure 6 gives dimensions for each elements and shows the connection used between the two structural layers. Typically, the rods of the gridshell are spaced by $300 \mathrm{~mm}$, with the five central arches separated by $150 \mathrm{~mm}$ only, which gives more rigidity to the principal loading directions. The rods are $10 \mathrm{~mm}$ in diameter and stacked directly on top of each other for a total thickness of the gridshell of $20 \mathrm{~mm}$. The target thickness for the concrete was $15 \mathrm{~mm}$. It is achieved using a small cap on top of the each connector as a visual cue: the concrete is flushed with the top surface of the cap when it is $15 \mathrm{~mm}$ thick. This guarantees a good control of the thickness at the connection. Despite the prestressing 
of the fiber glass mesh, some sagging is expected between the gridshell rods so that the concrete would be thicker there. The connector itself is an M3x20 screw, topped by the small cap to reach a total height of $25 \mathrm{~mm}$ below the screw head. The connector goes through $\varnothing 3.5 \mathrm{~mm}$ holes in the rods, and ties two intersecting rods together with a wing nut. The fiberglass mesh formwork is attached at each connector, between the top rod and the wing nut.

\subsection{Mix design of the fiber reinforced concrete}

The concrete mix used for the prototype was formulated especially for this application. It had to be easy to place on the formwork, and to remain on inclined surfaces without slipping. Due to the reduced thickness $(15 \mathrm{~mm})$, reinforcement could only be done by incorporating fibers in the fresh concrete. Therefore at the fresh state, the concrete must have a Bingham fluid type behavior, which is a fresh concrete described by a shear threshold and a dynamic viscosity [31]. Here we sought to obtain a high shear threshold that equilibrates shear stresses on inclined surfaces and low dynamic viscosity which facilitates the placing operations.

These specifications have led to the following guidelines:

- Small aggregates $\left(D_{\max } \leq 4 \mathrm{~mm}\right)$. The concrete is in fact a mortar.

- Water to cement ratio not too low $(e / c \geq 0.40)$ to minimize the dynamic viscosity.

- Incorporation of stainless steel fibers, the proportion of which was experimentally adjusted in order to obtain a satisfactory post-pic behavior in terms of ductility.

- Use of admixtures which improve sticking on lost formwork and diminish the risk of early age cracking.

There exist many different fiber types. Steel fibers tend to exhibit the best mechanical behavior for structural applications when compared to polymeric fibers. To ease the flushing of the concrete, soft stainless metallic fibers were selected, more precisely FibraFlex ${ }^{\circledR}$ fibers FF15E0/10 type, $15 \mathrm{~mm}$ length, from Saint-Gobain. Adjustment of the fiber content was completed experimentally.
Three concrete mixes representative of the various experiments are presented in Table 1. Mix A has a lower water content $(e / c=0.4)$ and was found hard to properly place. Mix B and Mix $\mathrm{C}$ have a higher water content $(e / c=0.5)$ and only differ by their fiber content. Structural testing on Mix B showed that this fiber content did not properly reinforce the concrete. Mix $\mathrm{C}$ is the mix used in the prototype. It fulfills all the placing and structural requirements. A mix with even more fiber content was tested and found to be not more resistant in bending than Mix C, but very difficult to place. Those mixes were tested in compression and flexion, using the standardized testing method EN 196-1 [32] with $4 \times 4 \times 16 \mathrm{~cm}$ samples, after 28 days curing time.

Table 1: Concrete mixes tested for the prototype. Composition given for $0.5 \mathrm{~L}$ of concrete, characteristics after 28 days.

\begin{tabular}{|l|c|c|c|}
\hline Components [g] & Mix A & Mix B & Mix C \\
\hline $\begin{array}{l}\text { Cement CEM I } \\
\text { 52.5MPa }\end{array}$ & 1000 & 1000 & 1000 \\
\hline Sand 0-4 mm, dry & 3242 & 2913 & 2913 \\
\hline Water eff. Content & 400 & 500 & 500 \\
\hline $\begin{array}{l}\text { Plasticizer Lafarge } \\
\text { F2 }\end{array}$ & 20 & 8 & 7 \\
\hline Latex SikaLatex & 30 & 30 & 30 \\
\hline FibraFlex FF/15E0 & 100 & 100 & 200 \\
\hline Property [MPa] & Mix A & Mix B & Mix C \\
\hline Flexural strength & 10 & 11 & 17 \\
\hline Compressive strength & 19 & 40 & 42 \\
\hline
\end{tabular}

\subsection{Construction}

The most significant steps of the construction process are presented in Figure 8. In addition, two short films have been made showing the construction process, and can be found at thinkshell.fr/hybridstructural-skin/. In chronological order, the construction steps are detailed below:

1. (Not shown) Prepare the rods for the gridshell: cut the rods at the required length and drill the holes that will receive the connectors at a constant step-size.

2. Assemble the flat grid on the ground. This is unskilled work that consists in bolting together the rods at each node.

3. Fix the fabric formwork on the flat gridshell. The chosen fabric is essentially a Chebyshev net like the grid, but with a much smaller cell size. If they 
initially share the same directions, the fabric will follow the grid during its elastic deformation. The formwork is hence laid on the flat grid, which spares the difficult task of patterning and fitting the fabric on a curved surface. The edges of the fabric are sewn into straight sheaths to be able to prestress it in the direction of the grid before deformation and to reduce wrinkling in the most sheared area and sagging under the load of the wet concrete.

4. Deform this assembly and fix each rod end on the contour. Except at the corner of the base square where supports are permanent, the ground connections are very simple and require little additional work.

5. Brace the gridshell with Polypropylene twine in the two diagonal directions. This bracing has a provisory function until the concrete has cured, so that the moderate stiffness of PP and ease of use for tying was a good compromise.

6. Prepare the formwork with a quick-setting cement layer. This intends at reinforcing the fabric in form in order to prevent the sagging between the rods. Because in our prototype this layer is visible on the underside, a large amount of time was spent in the finishing and smoothing.

7. Pour the concrete. The concrete was prepared and poured in three batches of $50 \mathrm{~L}$ each, for a total mass of concrete on the structure of $348 \mathrm{~kg}$, and average thickness of $15.4 \mathrm{~mm}$.

8. Cut away the temporary parts of the gridshell after the concrete cured seven days.

9. Protect the concrete skin with lime whitewash.

The man-hour costs of those steps are presented in Table 2 (all tasks were realized by the authors and students from the laboratory so that these durations must be considered as significantly larger than what could be achieved by skilled workers). The construction of the wooden base on wheels represents a large part of this time, but would not be needed in a larger project where the structure is fixed to the ground. The preparation of the gridshell is also time-consuming because it is a precise work where 500 holes must be drilled in circular rods at a constant distance with a constant axis orientation; it could easily be automated in a larger project. The next lengthy operation is the setting of the layer of quick-setting cement, for which a lot of care had to be taken on the smoothing of the underside (see Figure 7). To avoid this time consuming operation, more technical fabric dedicated to concrete formwork could be used as in [33] but it is not clear if those fabrics would be suitable here, where very low shear stiffness is needed as well as high stiffness in the main directions. Another option would be to keep this kind of fabric but to use a resin with a room-temperature cure instead of cement and to set it by projection.

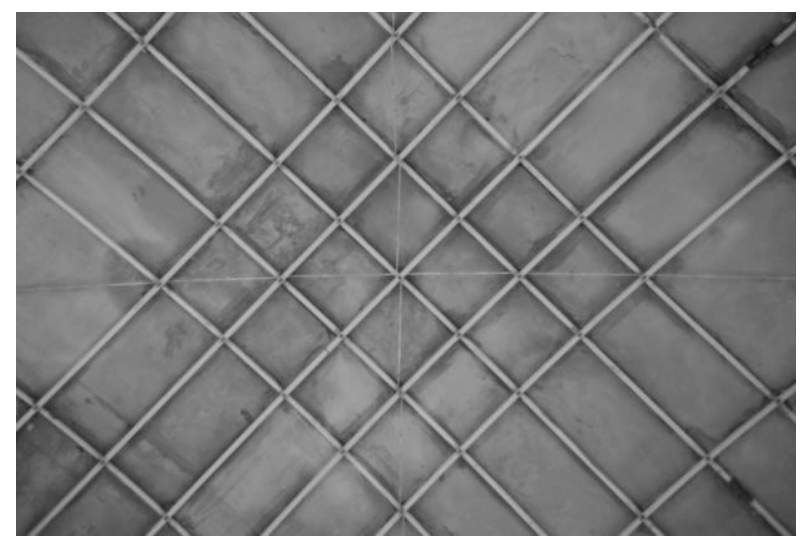

Figure 7: Inside view of the apex showing the smooth finish of the quick-setting cement.

Table 2: Labor costs involved in the construction.

\begin{tabular}{|l|c|}
\hline Activity & Time spent [hours] \\
\hline Build wooden base & 80 \\
\hline Prepare and assemble grid and connectors & 70 \\
\hline Prepare and install mesh formwork & 21 \\
\hline Erect and brace gridshell & 9 \\
\hline Place quick-setting cement & 40 \\
\hline Place concrete & 24 \\
\hline Remove temporary parts & 2 \\
\hline
\end{tabular}




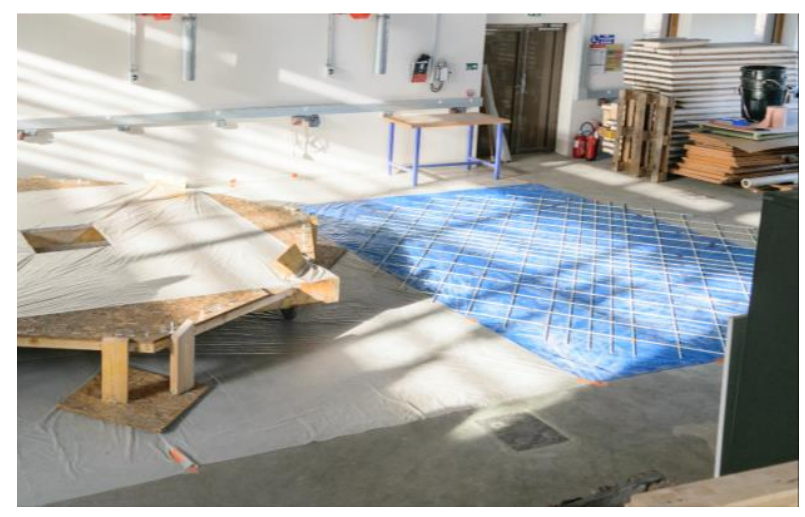

1. Gridshell preparation

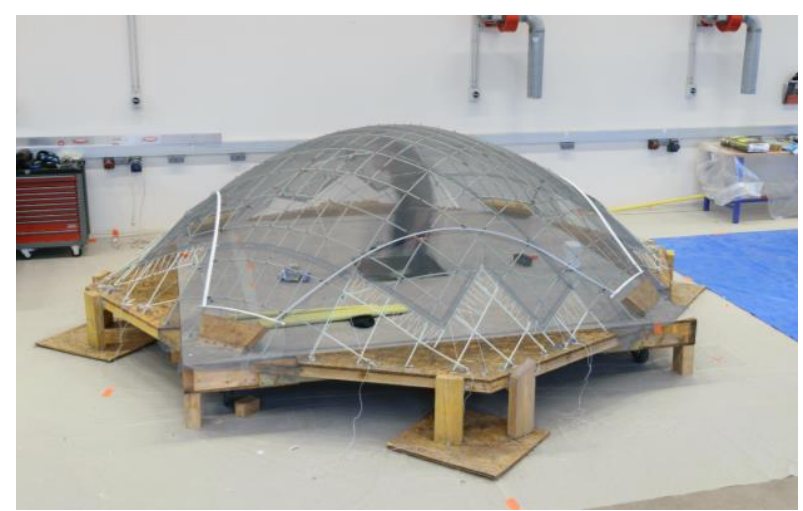

3. Gridshell erection and bracing

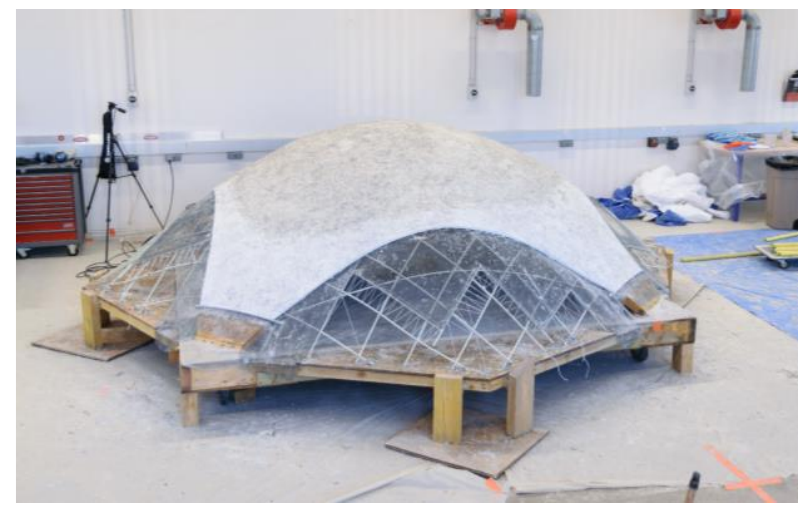

5. Concrete placing

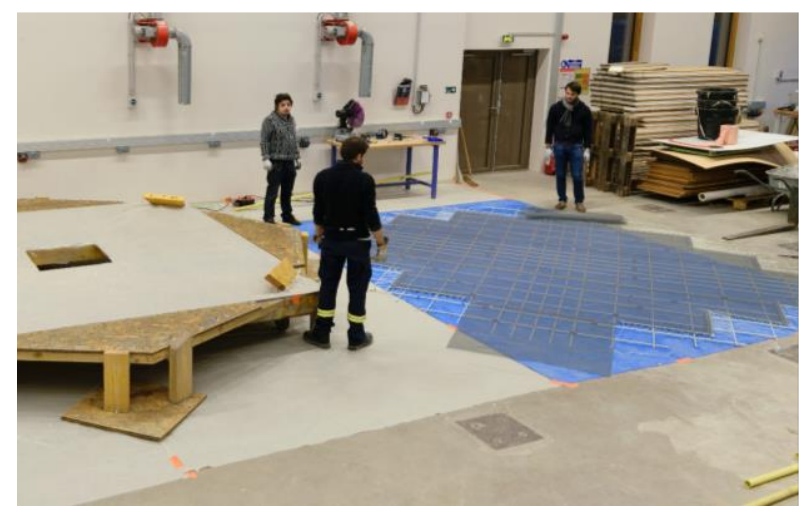

2. Mesh formwork preparation

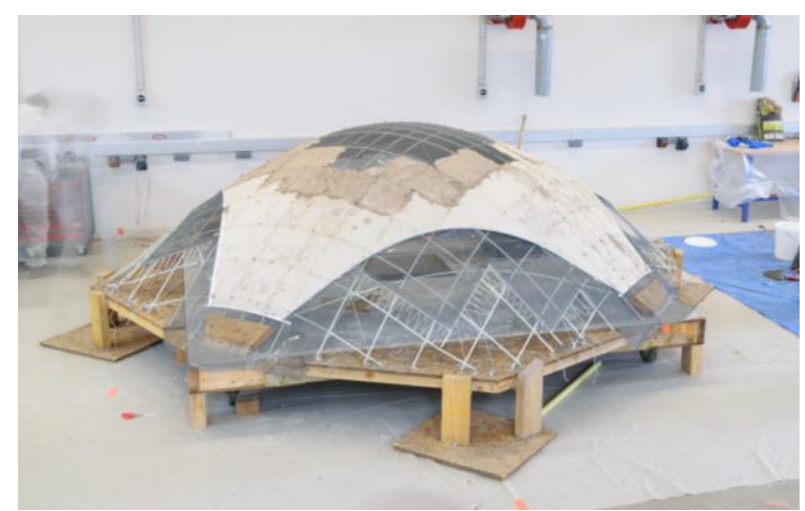

4. Quick-setting cement placing

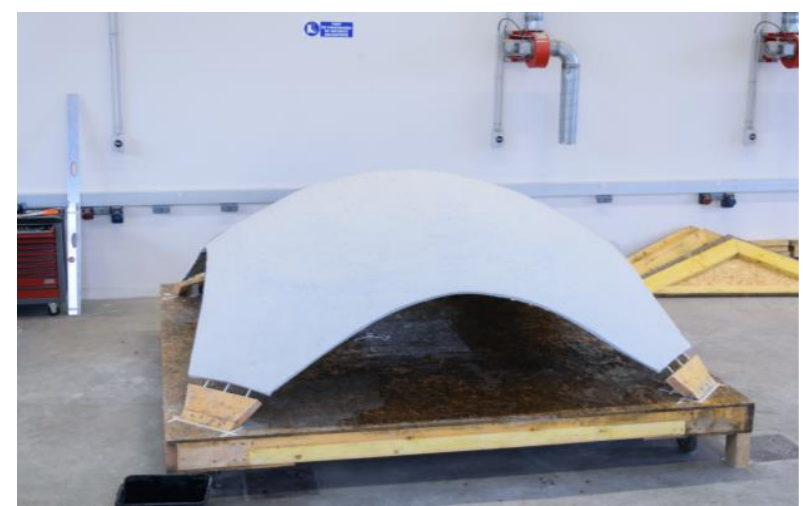

6. Finished structure, temporary parts removed

Figure 8: Steps of the construction process.

\subsection{Construction materials and cost}

The descriptions and costs of the materials used for the prototype are compiled in Table 3 . The wooden base with its rolling display has by far the largest cost with a share of $63 \%$, but this cost could be regarded as that of foundations and floor and therefore be considered external cost for the structure. The rest of the cost is shared by the gridshell $(46 \%)$, the formwork (29\%) and the concrete (25\%). The total cost per projected area is $151 € / \mathrm{m}^{2}$ with the base, $56 € / \mathrm{m}^{2}$ excluding the base. For comparison, the structure in [20], excluding the cost of the concrete, cost $162 \mathrm{CHF} / \mathrm{m}^{2}$ (or $133 € / \mathrm{m}^{2}$ in April 2013, when it was built), with almost $60 \%$ of the cost going to the wooden frame which is here truly part of the structure as a cable net cannot stand without the surrounding superstructures. 
Table 3: Cost of materials used for the construction of the prototype. Some materials where not directly paid for, their approximate retail price is given and noted with*.

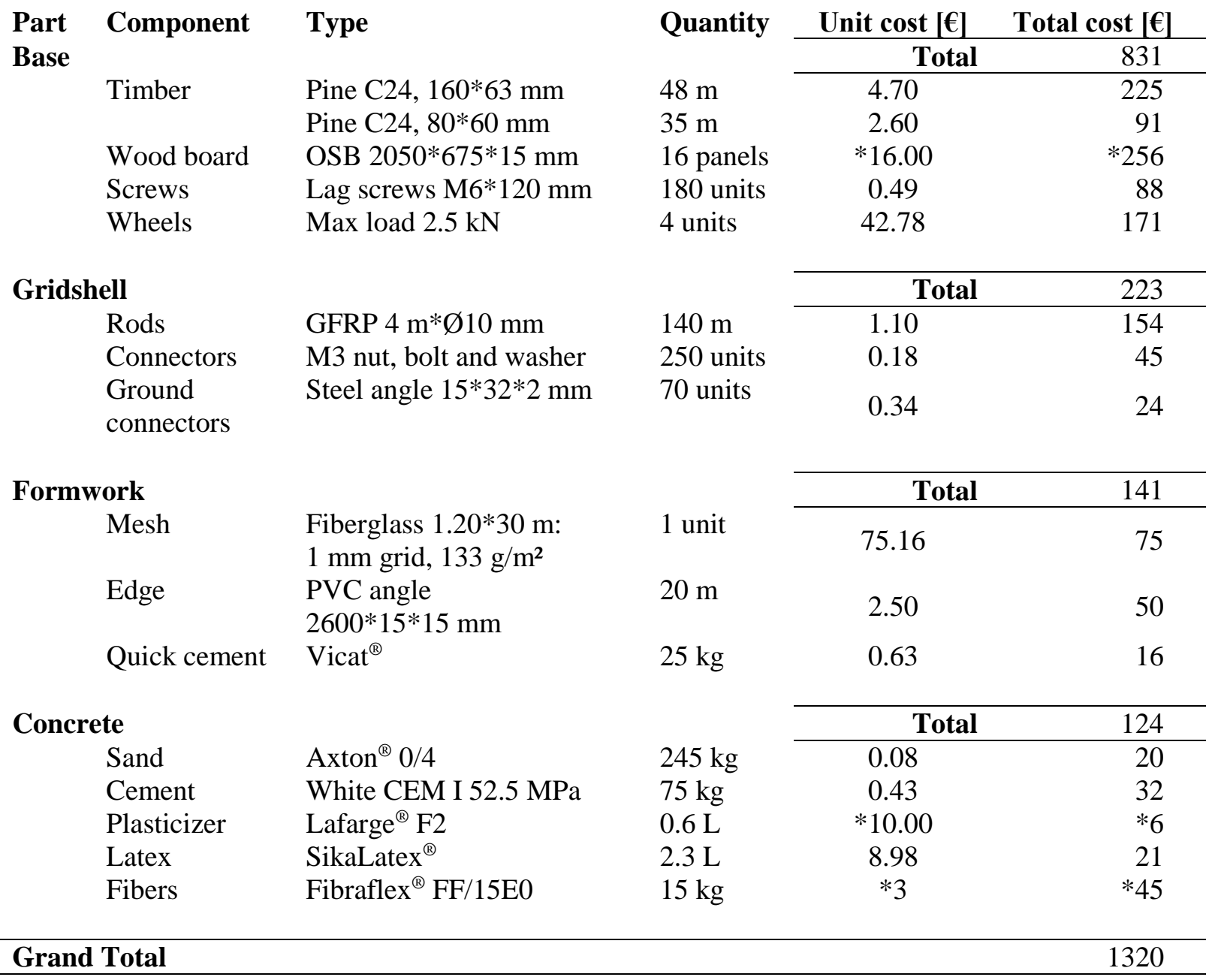

\section{DISCUSSION}

\subsection{Evaluation of the built prototype}

Here we investigated a novel type of shell where a GFRP elastic gridshell is associated to a fiber reinforced concrete envelope to achieve a composite behavior. The objective was twofold: first to enlarge the field of application of elastic gridshells by simplifying their bracing and their covering and second to renew the construction technique of thin concrete shells by the usage of an innovative formwork. Furthermore, the concept of a hybrid structural shell described here aims at reducing the self-weight of the structure to a minimum where the esthetical and mechanical performances of each material are used for the best. The formal universe is that of elastic gridshells, whose manifesto remains the Mannheim gridshell [4].

\subsection{Extrapolation to larger scale}

The built prototype proved the feasibility of the technique and the economic data provided shows that it has good potential for application to larger scales. For medium scale, say for cupolas between $10 \mathrm{~m}^{2}$ and $50 \mathrm{~m}^{2}$, the proposed technique can be directly adapted and implemented by local craftsmen (like for the Pishwanton gridshell construction [34]). The diameter of the gridshell members and of the connectors (which for the built prototype were slightly undersized) will have to be increased, but the size of the grid and its mass will remain low (about a hundred kilos) so that no extraordinary equipment will be needed for the forming of the grid. No cutting pattern is required for the fabric formwork which is flat, as is the grid: its manufacturing will remain simple, even if the material must be adapted to the scaling and changed into a more technical textile. 
Then the not too low water to cement ratio of the concrete makes it relatively easy to mix and to place.

For larger cupolas, single layer GFRP gridshells might be suited for spans until 15-20 m for which a construction process similar to that of Créteil structures remains applicable. For larger spans, double layer grids would be necessary which is still technically feasible but leads to increased complexity of the manufacturing and assembling. Then to keep the weight of the cover as low as possible it is mandatory to optimize the thickness of the concrete skin. To this end, it should be designed to resist local punching loads and distributed variable loads by bending between to members of the grid. Considering that the distance between members is about $1 \mathrm{~m}$, preliminary calculations with the proposed mix design showed that a thickness of $30 \mathrm{~mm}$ should be sufficient. Further development of this research program will thus focus on the improvement of the connection between the gridshell and the concrete envelope.

\section{ACKNOWLEDGMENTS}

The authors would like to thank all those who participated in the preparation, construction and testing of the prototype: M. Bonnet, M. Gillet and H. Orts for helping prepare the base and the gridshell; J-F. Caron, S. Gervilliers, V. Loing, P. Margerit, R. Mesnil, O. Perret and F. Tayeb for their help in the construction stages. This prototype would not have been standing today without them.

The authors acknowledge the sponsoring from SaintGobain who provided the FibraFlex steel fibers.

\section{REFERENCES}

[1] du Peloux, L., Tayeb, F., Baverel, O., and Caron, J.-F., Construction of a Large Composite Gridshell Structure: A Lightweight Structure Made with Pultruded Glass Fibre Reinforced Polymer Tubes, Struct. Eng. Int., 2016, 26 (2), pp.160-167(8). DOI: $10.2749 / 101686616 X 14555428758885$

[2] Baverel, O., Caron, J.-F., Tayeb, F., and du Peloux, L., Gridshells in composite materials: Construction of a $300 \mathrm{~m} 2$ forum for the Solidays' festival in Paris, Struct. Eng. Int., Vol. 22, No. 3, Aug. 2012, pp. 408-414. DOI: $10.2749 / 101686612 X 13363869853572$
[3] Wells, M., Taking shape: on site at Weald and Downland, RIBA J., Vol. 108, No. 4, 2001, pp. 75-76.

[4] Bundesgartenschau Mannheim 1975, Bauwelt, Vol. 66, No. 22, 1975.

[5] Ban, S., The Japanese pavilion, in Shigeru Ban, Ed. M. McQuaid, Phaedon, 2006, pp. 811.

[6] Otto, F., Multihalle Mannheim; the documentation on the design and execution work of Mannheim Hall. Mitteilungen des Institut für Leichte Flächentragwerke (IL) 13, Wittenborn Art Books, 1978.

[7] Harris, R., Rohmer, J., Kelly, O., and Johnson, S., Design and construction of the Downland Gridshell, Build. Res. Inf., Vol. 31, No. 6, 2003, pp. 427-454. DOI: $10.1080 / 0961321032000088007$.

[8] Harris, R., Haskins, S., and Roynon, J., The Savill Garden gridshell: Design and construction, Struct. Eng., Vol. 86, No. 17, 2008, pp. 27-34.

[9] du Peloux, L., Tayeb, F., and Lefevre, B., Formulation of a 4-DoF torsion/bending element for the formfinding of elastic gridshells, in Proc. IASS Symposium 2015, Eds. JB Obrębski and R. Tarczewski, p.1-14.

[10] Douthe, C., Caron, J.-F., and Baverel, O., Gridshell structures in glass fibre reinforced polymers, Constr. Build. Mater., Vol. 24, No. 9, 2010, pp. 1580-1589. DOI: 10.1016/j.conbuildmat.2010.02.037.

[11] Olcayto, R., Solutions: Timber Structures Gridshell glazes over the past, Building Design, Vol. 1776, pp. 14-17, 22-Jun-2007.

[12] Grafe, R., Gröbner, G., Grïndig, L., Hennicke, J., Matsushita, K., Otto, F., Sataka, K., Schaur, E., Schock, H.-J., and Shirayanagi, T., Grid shells. Mitteilungen des Institut für Leichte Flächentragwerke (IL) 10, Karl Krämer Verlag, 1974.

[13] Grafe et al, ibid., pp. 270-271.

[14] Grafe et al, ibid, pp. 308-315. 
[15] D'Amico, B., Kermani, A., Zhang, H., Pugnale, A., Colabella, S., and Pone, S., Timber gridshells: Numerical simulation, design and construction of a full scale structure, Structures, Vol. 3, Aug. 2015, pp. 227-235. DOI: 10.1016/j.istruc.2015.05.002.

[16] Mork, H. J., Dyvik, H. S., Manum, B., Rønnquist, A., and Labonnote, N., Introducing the segment lath - a simplified modular timber gridshell built in Trondheim Norway, in World Conference on Timber Engineering, 2016, pp. 1-8.

[17] Douthe, C., Mesnil, R., Orts, H., and Baverel, $\boldsymbol{O}$., New shapes for elastic gridshells covered by planar facets, in Proceedings of the IASS Annual Symposium, Eds. K. Kawaguchi, M. Ohsaki, and T. Takeuchi, 2016, pp. 1-9.

[18] Abdelgader, H., West, M., and Górski, J., State-of-the-art report on fabric formwork, in Proceedings of the International Conference on Construction and Building Technology, 2008, pp. 93-105.

[19] Orr, J. J., Ibell, T. J., Darby, A. P., and Evernden, M., Extraordinary possibilities using fabric to form concrete structures, in Proc. 5th Int. Conference on Structural Engineering, Mechanics and Computation, Ed. Z. Alphose, 2013, pp. 37-41. DOI: 10.1080/19373260.2013.831380.

[20] Veenendaal, D. and Block, P., Design process for prototype concrete shells using a hybrid cable-net and fabric formwork, Eng. Struct., Vol. 75, 2014, pp. 39-50. DOI: 10.1016/j.engstruct.2014.05.036.

[21] Peerdeman, B., Analysis of Thin Concrete Shells Revisited: Opportunities due to Innovations in Materials and Analysis Methods, Delft University of Technology, 2008.

[22] Farshad, M., Design and Analysis of Shell Structures, $\quad$ Vol. $16 . \quad$ Springer Science+Business Media, 1992. DOI: 10.1007/978-94-017-1227-9.

[23] Maurin, B. and Motro, R., Concrete Shells Form-Finding with Surface Stress Density
Method, J. Struct. Eng., Vol. 130, 2004, pp. 961-968. DOI: 10.1061/(ASCE)07339445(2004)130:6(961).

[24] Maurin, B. and Motro, $R$., The surface stress density method as a form-finding tool for tensile membranes, Eng. Struct., Vol. 20, No. 8, 1998, pp. 712-719. DOI: 10.1016/S01410296(97)00108-9.

[25] Grafe et al, ibid. pp. 138-155.

[26] Lefevre, B., Douthe, C., and Baverel, O., Buckling of elastic gridshells, J. IASS., Vol. 56, No. 3, 2015, pp. 153-171.

[27] Barnes, M. R., Form Finding and Analysis of Tension Structures by Dynamic Relaxation, Int. J. Sp. Struct., Vol. 14, No. 2, 1999, pp. 89-104. DOI: 10.1260/0266351991494722.

[28] Douthe, C., Baverel, O., Caron, J.-F., Formfinding of a grid shell in composite materials, J. IASS., Vol. 47, No. 150, 2006, pp. 53-62.

[29] Mesnil, R., Ochsendorf, J., and Douthe, C., Stability of Pseudo-Funicular Elastic Grid Shells, Int. J. Sp. Struct., Vol. 30, No. 1, 2015, pp. 27-36.

[30] Scordelis, A. C., Stability of reinforced concrete domes and hyperbolic paraboloid shells., in Concrete Shell Buckling, Vol. 67, Eds. EP Popov and SJ Medwadowski, American Concrete Inst., 1981, pp. 63-110.

[31] de Larrard F., Concrete mixture proportioning: a scientific approach. Modern Concrete Technology, E \& FN Spon, 1999.

[32] AFNOR, NF EN 196-1: Méthodes d'essais des ciments, 1995.

[33] Veenendaal, D., West, M., and Block, P., History and overview of fabric formwork: Using fabrics for concrete casting, Struct. Concr., Vol. 12, No. 3, 2011, pp. 164-177. DOI: $10.1002 /$ suco.201100014.

[34] Sassi, P., Case study: Community self-build, in Strategies for Sustainable Architecture, Taylor \& Francis, 2006, pp. 60-61. 